Neurosurg Focus 17 (2):E7, 2004 Click here to return to Table of Contents

\title{
Glomus jugulare tumors with intracranial extension
}

\author{
C. Gary Jackson, M.D., David M. Kaylie, M.D., George CopPit, M.D., \\ AND EDWARD K. GARDNER, M.D.
}

The Otology Group at Vanderbilt, Nashville, Tennessee

\begin{abstract}
Glomus tumors provide unique surgical challenges for both tumor resection and defect reconstruction. Tumors with intracranial extension compound these challenges. Surgical techniques have evolved, and now, with a multidisciplinary team, single-stage surgeries are the standard. In this paper the authors will report the results of the Otology Group protocol for surgical management of glomus tumors with intracranial extension. Particular attention will be paid to prevention of cerebrospinal fluid leaks with the use of vascularized tissue for defect reconstruction.
\end{abstract}

\section{KEY WORDS - glomus jugulare tumor • intracranial extension • skull base surgery • single-stage resection}

Glomus tumors are the second most common neurootological tumor after acoustic neuromas. The surgical management of these tumors has evolved in conjunction with the evolution of the specialty of skull base surgery. Microsurgical equipment and techniques have developed to the point where the question of surgical resectability is rarely an issue. Imaging technology has also improved and preoperative planning is sophisticated and exact. Nonetheless, neurootological skull base surgery for the resection of glomus jugulare tumors is not without risk and morbidity.

Glomus jugulare tumors arise in the lateral skull base and grow along the paths of least resistance (Fig. 1). They often will develop intracranial extension. They can gain access into the subarachnoid space by penetrating the dura of the posterior fossa, growing along cranial nerves (Fig. $2) .^{7}$ They can also, less commonly, penetrate the dura of the middle fossa. Their involvement with major vessels, proximity to cranial nerves, and their propensity for intracranial extension can result in significant morbidity from tumor resection. Kinney ${ }^{9}$ recommended a two-stage resection of glomus jugulare tumors with intracranial extension. He suggested that the intracranial component be removed first. Jenkins and Fisch ${ }^{8}$ recommended staged resection of tumors that have a $2-\mathrm{cm}$ or greater intracranial extension. Jackson, et al., ${ }^{7}$ showed that a single-stage resection and reconstruction offered the greatest likelihood of complete tumor removal while preserving local tissue for use in reconstruction.

Glomus jugulare tumors with intracranial extension have posed great surgical and reconstruction challenges.

\footnotetext{
Abbreviations used in this paper: $\mathrm{CA}=$ carotid artery; $\mathrm{CSF}=$ cerebrospinal fluid; $\mathrm{CT}=$ computerized tomography; $\mathrm{MR}=$ magnetic resonance.
}

These challenges have, in turn, led to the development of innovative surgical solutions. Glomus jugulare tumors grow in a manner that is highly variable from patient to patient. The variability of tumor presentation has necessitated the development of a creative multidisciplinary approach to manage these complicated lesions. In this report we will discuss the single-stage surgical and reconstructive management of glomus jugulare tumors that have intracranial extension.

\section{CLINICAL MATERIAL AND METHODS}

\section{Patient Population}

Between January 1, 1971, and December 31, 2003, 228 patients underwent surgery for glomus tumors at our institution. The mean age was 33 years and the age range was 12 to 58 years. The male/female ratio was $2.3: 1$. One hundred ninety-two patients had glomus jugulare tumors, 33 had glomus vagale lesions with skull base involvement, and three had carotid body tumors with skull base involvement.

\section{Diagnostic Methods}

Clinical findings alert the clinician to the possibility of a lateral skull base lesion with involvement of the ear. Symptoms usually include pulsatile tinnitus and hearing loss. Conductive hearing loss is the result of mechanical obstruction of the ossicular mechanism by tumor, whereas sensorineural hearing loss is a consequence of labyrinthine involvement. ${ }^{5} \mathrm{~A}$ vascular tumor is often seen in the inferior aspect of the middle ear. A glomus tympanicum tumor will sometimes have tumor margins that are visible for $360^{\circ}$, whereas the inferior border of a glomus jugulare tumor will not be visible. ${ }^{4}$ Lower cranial nerve 


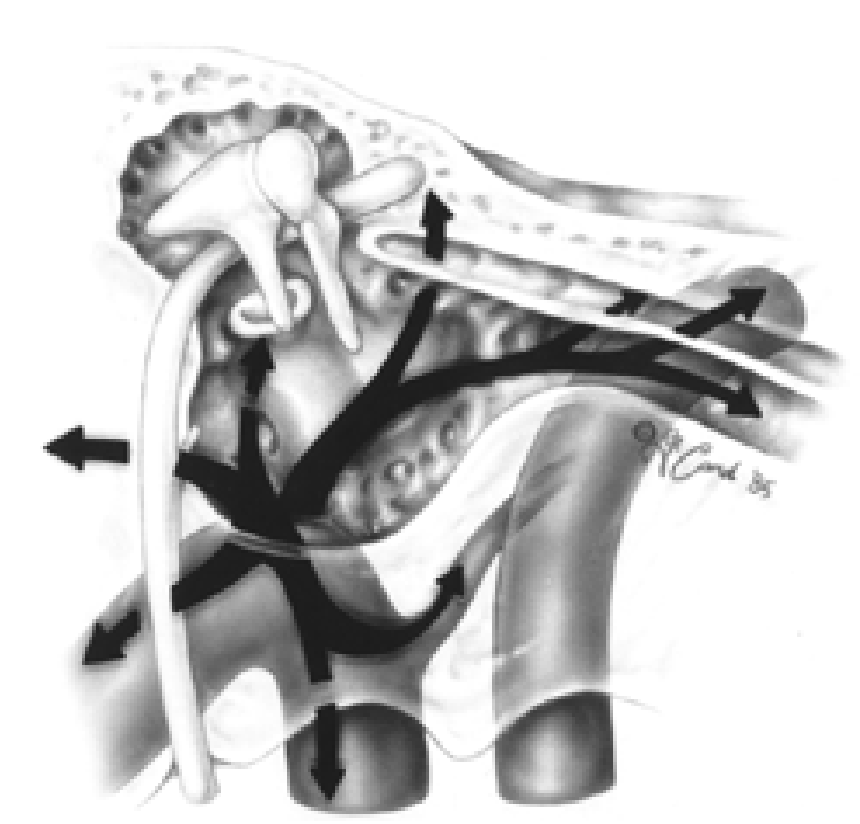

Fig. 1. Extent of glomus tumor is highly variable along lines of least resistance.

palsies can occur with tumor involvement of the pars nervosa. . "Idiopathic" vocal cord palsy or paralysis is an ominous sign indicating vagus nerve involvement. With the advancements in MR imaging, this diagnosis should be considered obsolete. A complete cranial nerve examination will elucidate the extent of pars nervosa involvement.

The goals of surgical planning are to determine the size, extent, and type of tumor (glomus tympanicum or jugulare), presence of associated lesions, and status of major blood vessels. Preoperative MR imaging is used to determine the type and extent of tumor. It can be used to deter-

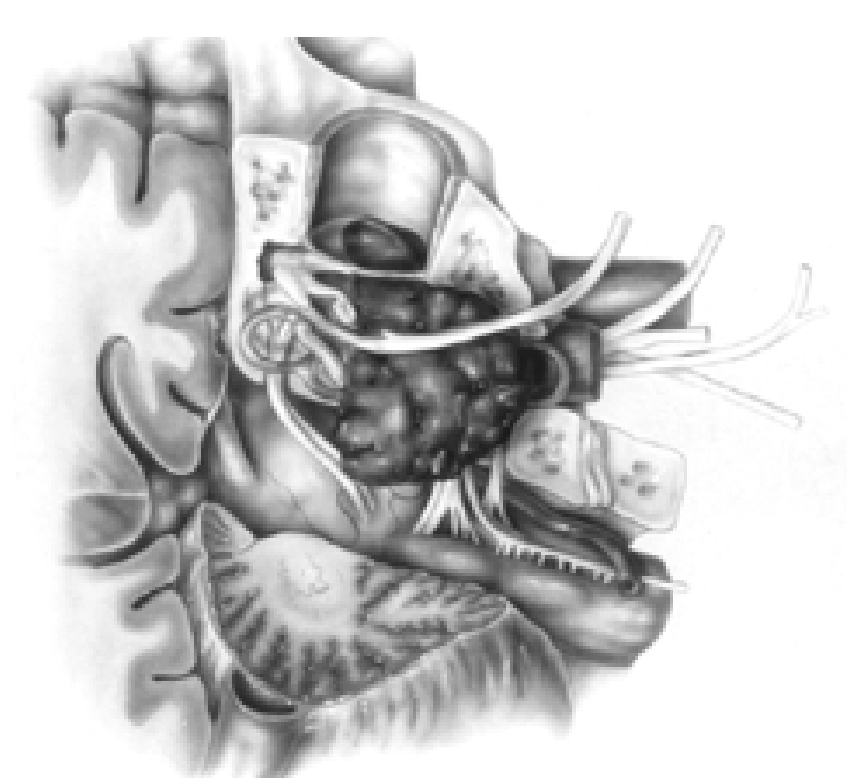

Fig. 2. Intracranial extension is seen when tumor enters into the subarachnoid space directly through dura or along cranial nerves. mine intracranial extension accurately in 95 to $98 \%$ of cases, ${ }^{6}$ and it provides information about the tumor's relation to the carotid region. A CT scan provides superior detail of bone involvement around the jugular bulb, carotid canal, and inner ear. ${ }^{5}$ The axial and coronal neuroimages will reveal bone erosion of the jugular foramen and inner ear. Imaging of only the temporal bone is insufficient. Synchronous lesions are seen in $10 \%$ of patients with glomus tumors and $50 \%$ of patients with hereditary paragangliomas. ${ }^{2}$ Therefore, MR imaging of the neck is mandatory. Angiography is performed in all patients the day before surgery. This provides information about patency of the major vessels and the tumor's relationship to these vessels. It also allows for preoperative embolization of the tumor. Embolization should be performed within 72 hours of surgery, and preferably within 24 hours. ${ }^{4}$

Paragangliomas have the potential for secreting catecholamines and neuropeptides. ${ }^{2}$ Approximately 1 to $3 \%$ of all paragangliomas are secretors and are considered functional tumors. ${ }^{4}$ The evaluation of a patient with a glomus tumor should specifically seek symptoms indicative of catecholamine production, such as palpitations, excessive sweating, and headaches. All patients should be screened with blood tests for elevated catecholamine metabolites. Patients who have "functioning" tumors need to receive both alpha and beta blockers preoperatively. ${ }^{2}$ Paragangliomas have been associated with other neoplasms such as pheochromocytoma, thyroid neoplasms, parathyroid adenomas, and multiple endocrine neoplasm syndromes; ${ }^{4}$ these need to be ruled out.

\section{Surgical Approach}

Surgical approaches to the cranial base are quite complex, requiring the expertise of practitioners from several disciplines, ${ }^{7}$ including neurosurgeons, neurootological surgeons, and head and neck reconstructive surgeons. The various approaches have been described in great detail elsewhere (Fig. 3). ${ }^{3}$ The basic surgical principle of wide exposure is particularly important for tumors with intracranial extension. The exposure afforded by the pre-

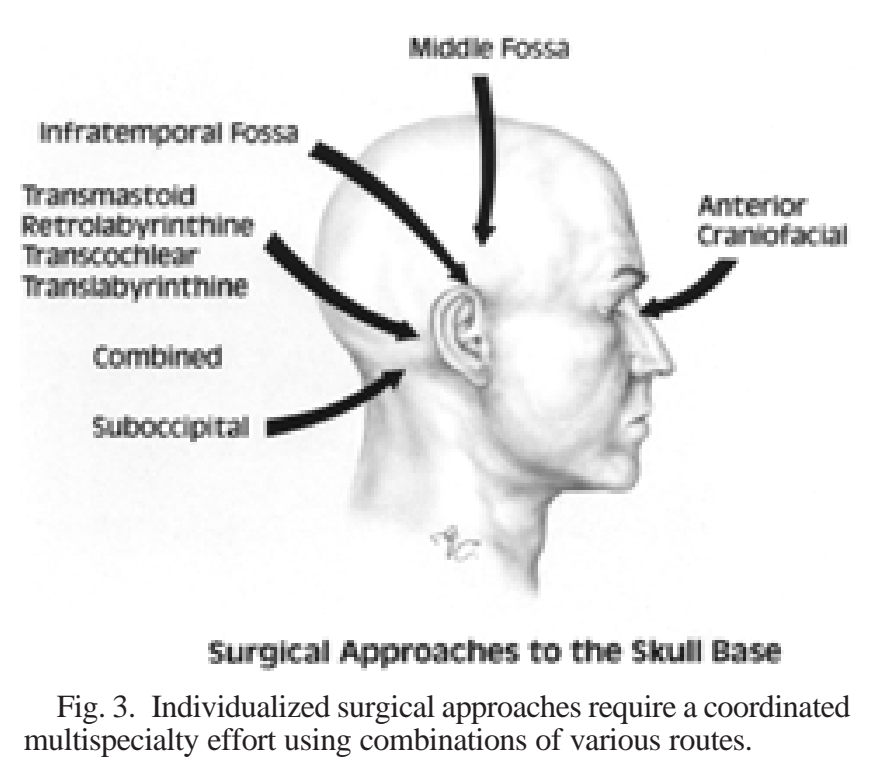


viously described bone dissection is extensive and will provide access to margins of the entire tumor and all vital vascular, neural, and intracranial structures (Fig. 4). Extension of the dissection for greater access to the posterior fossa is easily accomplished. The dissection can easily be enlarged to expose posterior or middle cranial fossae.

The surgery proceeds in an orderly sequence, with attention paid initially to identification of the CA proximally in the neck and distally in the temporal bone. Proximal and distal control of major vessels is a cardinal principle of vascular surgery and holds true for glomus tumor surgery. The level of distal control is dictated by the extent of the tumor, which may necessitate dissection of the tympanic, petrous, or cavernous portion of the artery. It is critical to have $180^{\circ}$ exposure of the CA to allow for instrumentation in the event of loss of integrity or resection of the artery. ${ }^{3}$

Facial nerve management options for tumors with intracranial extension require some degree of facial nerve mobilization (Fig. 5). ${ }^{4}$ It is not possible to expose the jugular bulb adequately without mobilizing the facial nerve. A short mobilization extends from the external genu of the facial nerve to the parotid, and patients generally have excellent long-term facial function thereafter. ${ }^{4}$ Larger tumors require a long mobilization from the internal genu laterally. Transient facial paralysis will occur, but long-term outcome is generally very good, resulting in House-Brackmann Grades II to IV. ${ }^{4}$ Resection of the facial nerve is rarely necessary, but is unavoidable if the nerve is infiltrated by the tumor and dissection is not possible. Preoperative facial paralysis invariably is caused by tumor infiltration into the facial nerve. Exposure of the petrous $\mathrm{CA}$ and infratemporal fossa requires anterior-inferior dislocation of the mandible. This can only be safely done with mobilization of the facial nerve. ${ }^{3}$

Lower cranial nerve preservation is facilitated at this point by identification of the nerves in the neck. They can be dissected from the neck, where they are uninvolved with tumor, into the pars nervosa, and intracranially if necessary. This technique provides maximum safety and nerve preservation. The intracranial extension compo-

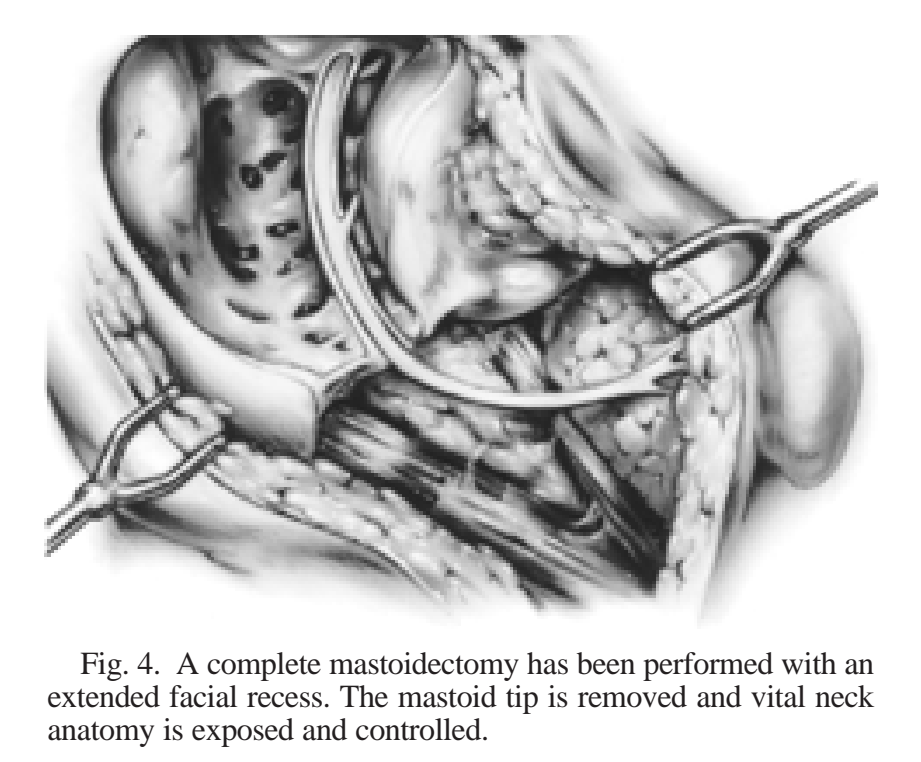

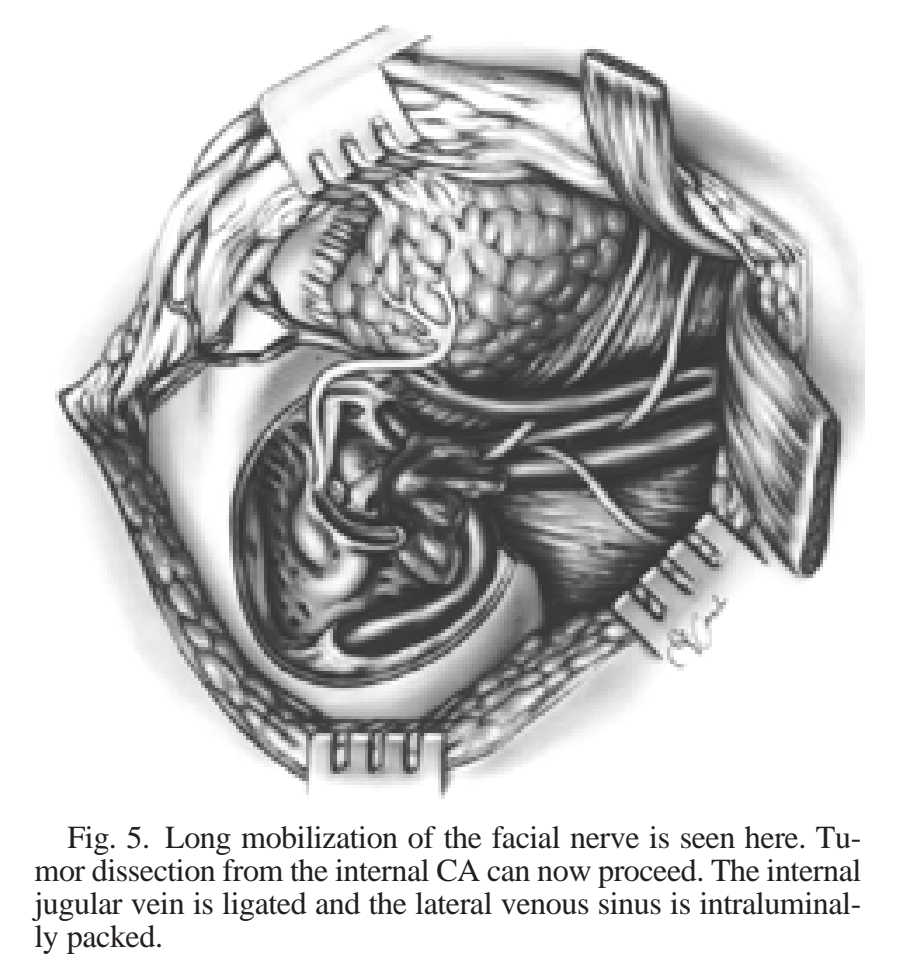

nent of the tumor is then removed. When there is more extensive intracranial involvement, a larger posterior cranial fossa exposure may be necessary. This is easily accomplished; the prior bone dissection has afforded easy access for a posterior fossa craniotomy. Removal of tumor from the posterior fossa is rarely difficult because the tumor has been largely devascularized by this point in the surgery (Figs. 6 and 7). ${ }^{6}$

\section{Reconstruction of Defects}

Dural defects are the consequence of resection of tumors with intracranial extension. Because of this, preven-

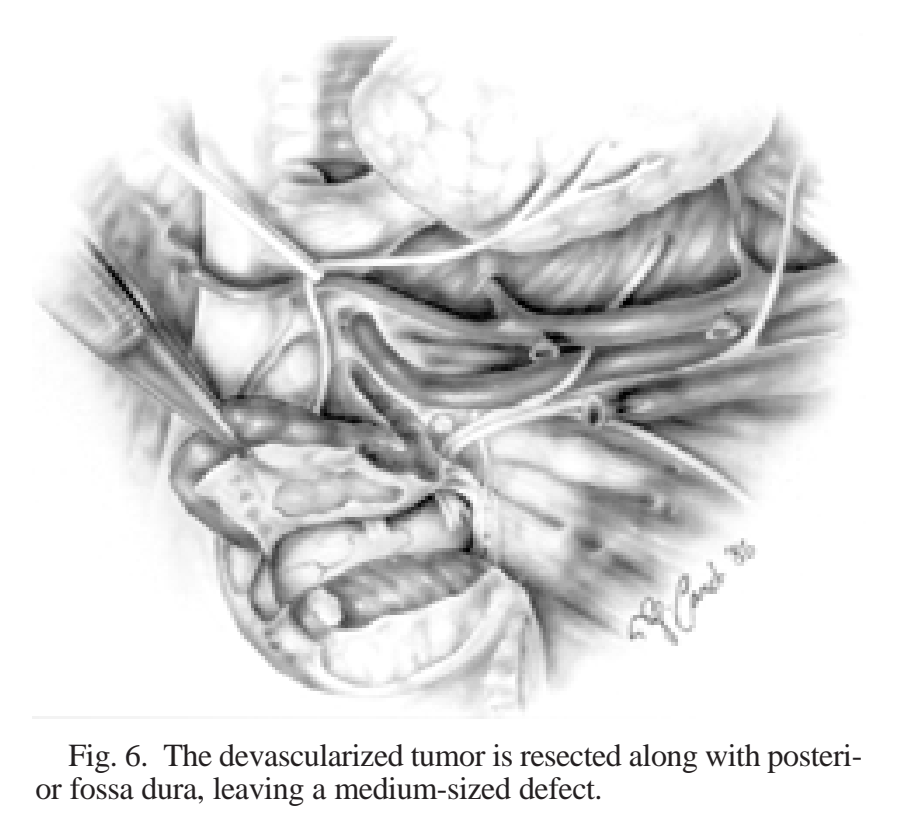




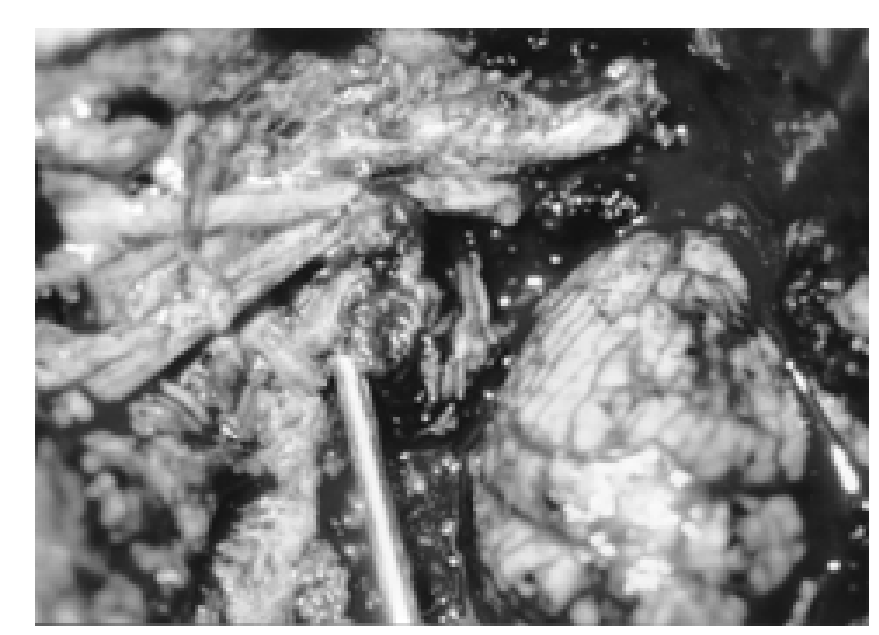

Fig. 7. The cerebellum is exposed in this medium-sized defect.

tion of CSF leaks is a particular challenge. Dural defect reconstruction is size-dependent and the complexity of the reconstruction should be tailored to the size of the defect. ${ }^{5}$ Basic principles of defect reconstruction include exclusive use of vascularized tissue supported by tissue bulk to resist CSF pressure and to support the dural reconstruction. Lumbar drainage is uniformly used to decompress the CSF space. ${ }^{4}$

Successful defect reconstruction is dependent on the careful preservation and mobilization of tissues. The initial incisions are designed to provide the necessary surgical exposure, but equally important, they are designed to allow for reconstruction and closure (Fig. 8). ${ }^{6}$ The workhorse of reconstructive flaps is the superficial temporalis fascia flap. Its design begins with the initial skin incision described by Netterville and Civantos (Figs. 9 and 10). ${ }^{10}$ It is based on the anatomical description of the blood supply

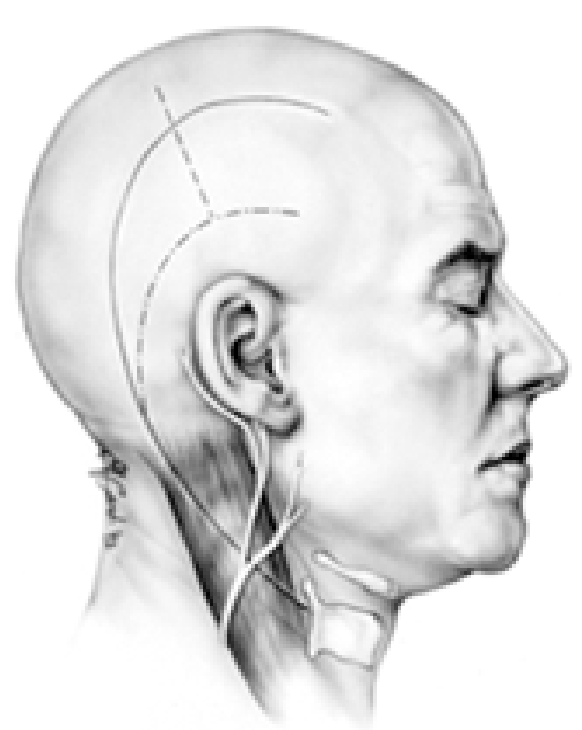

Fig. 8. The large $\mathrm{C}$-shaped incision is outlined superior enough to prevent foreshortening of the superficial temporalis fascia flap. The incision is now modified to the smaller C-shaped incision. to this tissue by Abu-Hassan, et al. ${ }^{1}$ Supportive tissue is necessary to provide bulk for cosmetic purposes as well as supporting dural reconstruction and internal CA coverage. Free abdominal fat works well. The sternocleidomastoid muscle flap is closed over the reconstruction to hold the fat packing firmly in place with pressure on the dural defect. This closure is used regardless of the presence or absence of the external auditory canal (Fig. 11). Lumbar drainage is used for 5 to 7 days to allow for adequate tissue healing. ${ }^{6}$

Small defects are usually seen when the labyrinth is left intact ${ }^{7}$ and the intracranial extension of tumor involves the pars nervosa (Fig. 12). ${ }^{3}$ This size defect is amenable to reconstruction with the vascularized superficial temporalis fascia flap, placement of abdominal fat, and primary wound closure. ${ }^{6}$ Medium-sized defects result from more extensive temporal bone dissection and larger dural resections (Fig. 13). Local tissue may not be available in these cases because of previous surgeries or radiation. In these instances, larger myocutaneous flaps like the trapezius or the latissimus dorsi flaps are used. ${ }^{10}$ Lumbar drains are always used postoperatively. Large defects are rare, and the special needs that arise from such defects are individually addressed. Prior surgery, extensive CA exposure, or prior radiation can all result in large dural defects. Microvascular free flaps can be used when local tissue is not available and the defect is very large. The rectus abdominis flap is suitable because it provides vascularized tissue and bulk.

\section{RESULTS}

The reconstruction protocol changed substantially in 1987 , and the results prior to this have been previously reported..$^{10}$ The following results are derived from surgeries for tumors with intracranial extension and for those resulting in dural defects between January 1, 1987, and December 31, 2003.

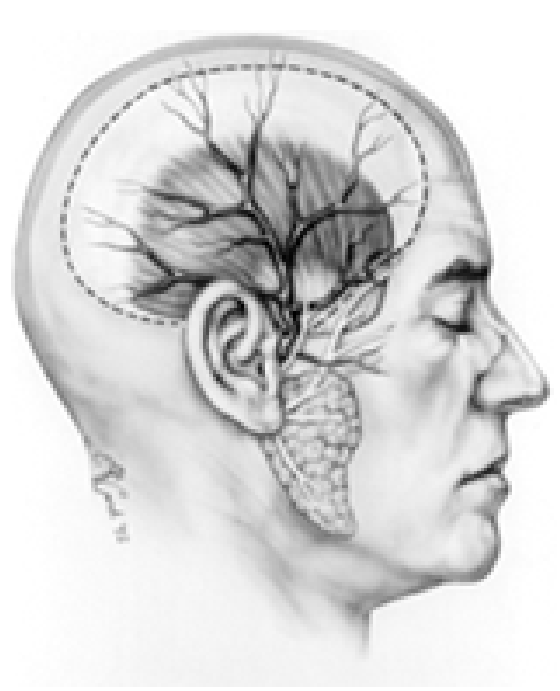

Fig. 9. The superficial temporalis fascia flap has great size potential. This flap is supplied by the superficial temporal artery. The smaller deep temporalis fascia is supplied by the middle temporal artery. 


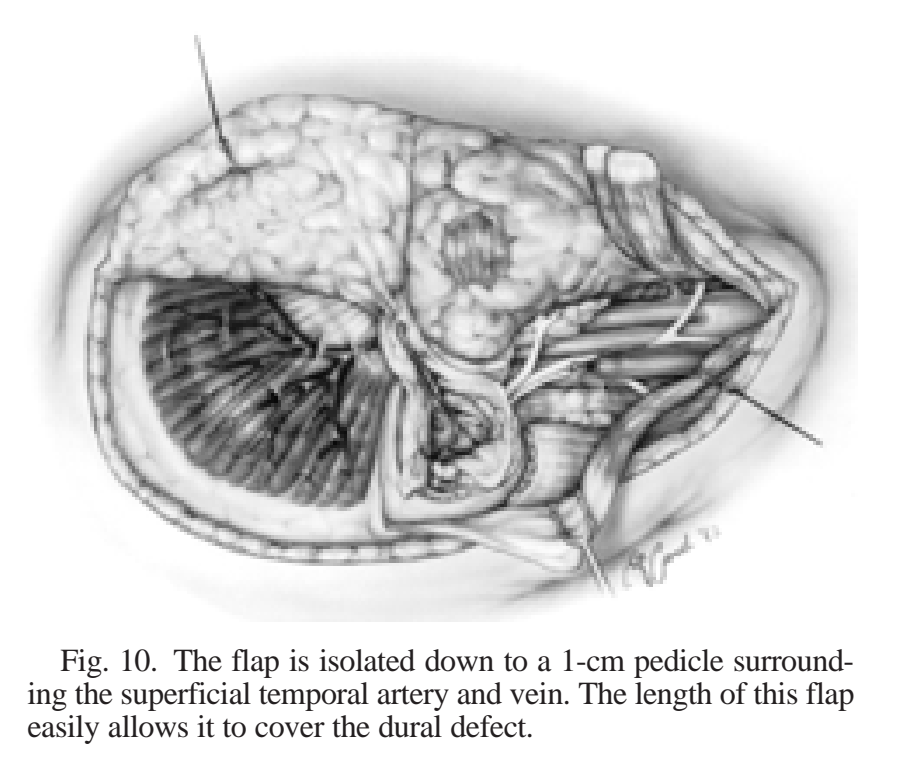

Twenty-two patients had tumors with intracranial extension and 29 had tumors resulting in dural defects without intracranial extension. The tumors with intracranial extension ranged from 1.5 to $7 \mathrm{~cm}$, with a mean size of $4.3 \mathrm{~cm}$. The intracranial component ranged from 0.3 to $4 \mathrm{~cm}$ and its mean size was $1.6 \mathrm{~cm}$. Tumors with dural defects but no intracranial extension ranged from 1.9 to 5 $\mathrm{cm}$, with a mean size of $3.4 \mathrm{~cm}$. Multicentric tumors were seen in $9 \%$ of all patients. Subtotal resection occurred in $11 \%$ of patients with intracranial extension.

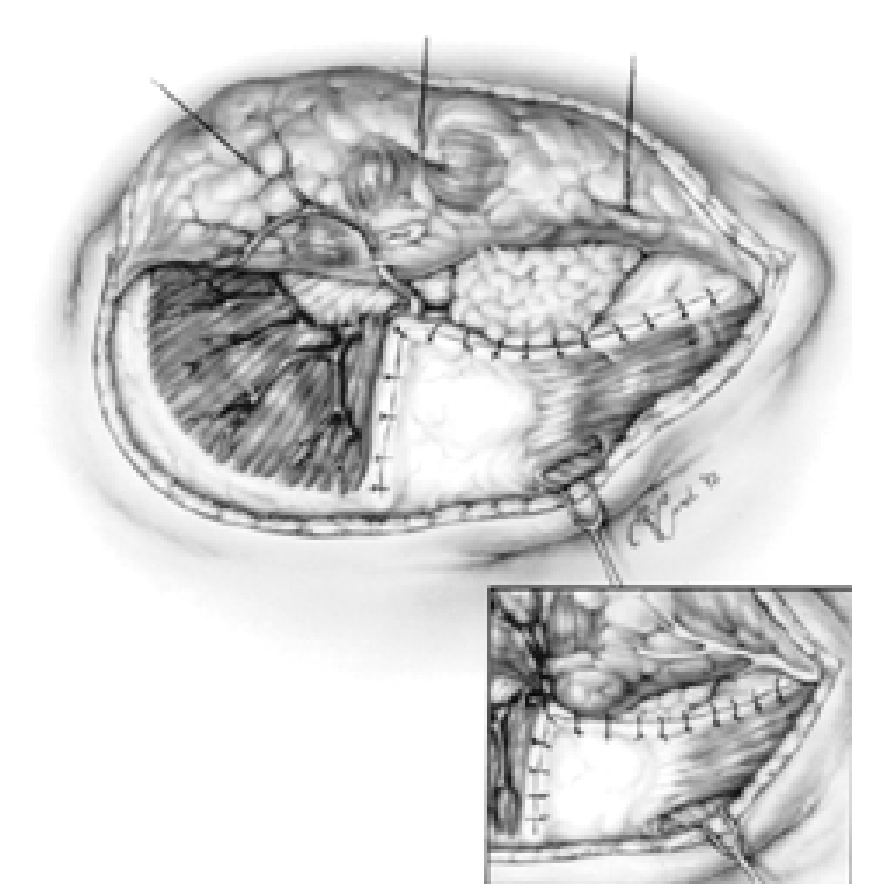

Fig. 11. The sternocleidomastoid muscle facial flap is closed over the free fat graft to the deep temporal fascia as well as the temporalis muscle. This forms a tight closure and firmly holds the fat in place with pressure on the dural defect.

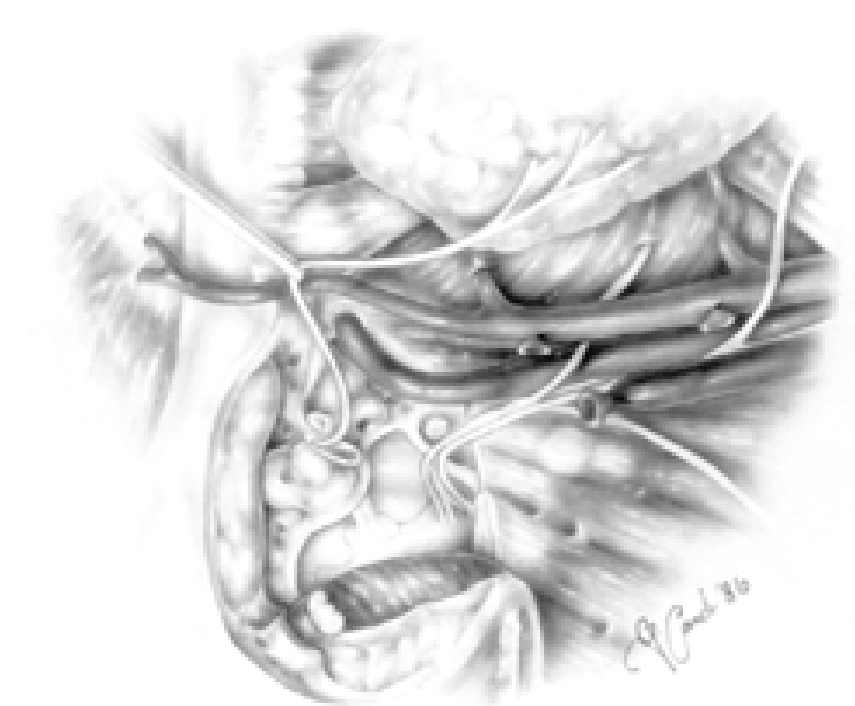

Fig. 12. The small dural defect seen here is based on the pars nervosa.

Follow-up duration ranged from 9 to 76 months (mean 36 months). Sixteen patients required a dural patch in addition to the surgical defect reconstruction. All patients underwent lumbar drainage for 5 to 7 days. Other complications included aspiration in seven patients, abscess and meningitis in two each, and pseudomeningocele, first bite, and skin necrosis in one each. A CSF leak was seen in three patients (6\%), one of whom had CSF rhinorrhea, which was fixed by oversewing the eustachian tube. The

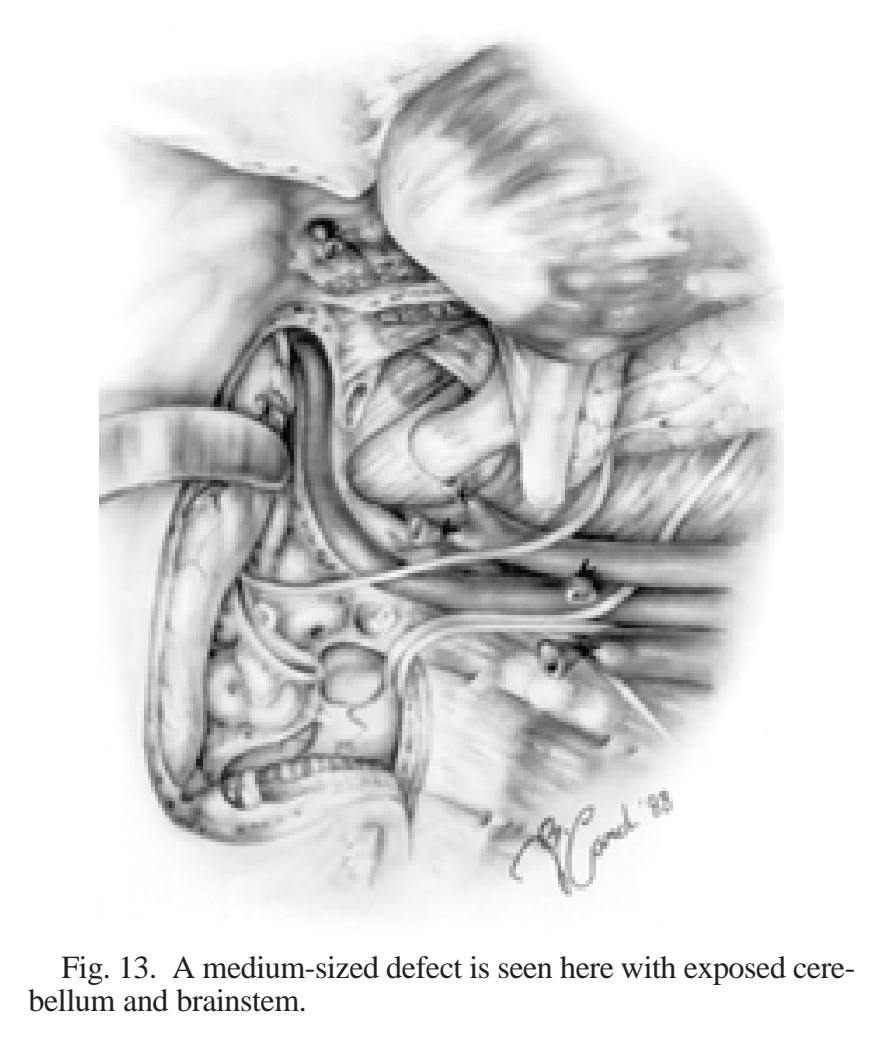


other two patients had CSF collections in the neck, one as the result of a wound abscess. There were no deaths in this group.

\section{DISCUSSION}

Defect reconstruction after removal of glomus jugulare lesions will often present challenges that are not seen in other neurootological procedures such as acoustic neuroma surgeries. The temporal bone dissection in acoustic neuroma surgeries is often less extensive than in glomus jugulare resections. Local tissues are often compromised in glomus tumor resection by prior radiation, embolization, or prior surgery, but are readily available in acoustic neuroma surgery. Glomus tumor surgeries also result in increased CSF pressure because of occlusion of the dural venous sinuses, which requires innovative CSF leak prevention strategies. The $\mathrm{CA}$ is exposed in all surgeries of glomus tumor with intracranial extension, which is not the case in acoustic neuroma surgery. For these reasons, a comprehensive, multidisciplinary strategy for managing these tumors has been developed.

Glomus tumors with intracranial extension are best resected in a single stage. This can only be achieved when the entire tumor and vital structures are adequately exposed. The surgical team consists of a head and neck/reconstructive surgeon, a neurootological/skull base surgeon, and a neurosurgeon. If this team cannot be assembled, a staged procedure should then be executed..$^{10}$ These surgeries take approximately 8 to 12 hours with an experienced surgical team. If the surgery cannot be completed in a reasonable amount of time, a staged procedure should be considered. Subtotal resection is also necessary in some patients. In $11 \%$ of surgeries in this series, complete resections were not possible. This is usually because of tumor adherence to brainstem, involvement with the cavernous sinus, or patients' wishes to preserve cranial nerves if they are involved with tumor. Proper imaging includes CT and MR studies, which are used to determine the size, extent, and intracranial extension of the tumor. ${ }^{5}$

The methods described here are based on the concept of defect reconstruction with vascularized tissue at minimized CSF pressure. The evolution of these strategies resulted in reduced CSF leakage rates. The current protocol has been in place since 1987. Before that, $19 \%$ of patients with intracranial extension experienced CSF leaks, ${ }^{10}$ whereas in this series only three patients had a CSF leak. The CSF dynamics are altered in the perioperative period, and lumbar drains, although not without risks, are a manageable solution for single-stage glomus tumor resection and defect reconstruction. ${ }^{7}$

\section{CONCLUSIONS}

Skull base surgery has evolved in complexity and elegance. A wide range of surgical and reconstructive options exists for a wide range of tumors. Preoperative planning with a multidisciplinary team allows for an operation that is exactly tailored to the individual patient's needs. When these principles are applied, functional outcome and the chance of tumor cure improve while complications decrease.

\section{References}

1. Abul-Hassan HS, von Drasek Ascher G, Acland RD: Surgical anatomy and blood supply of the fascial layers of the temporal region. Plast Reconstr Surg 77:17-28, 1986

2. Gulya AJ: The glomus tumor and its biology. Laryngoscope 103 (Suppl 60):7-15, 1993

3. Jackson CG: Basic surgical principles of neurotologic skull base surgery. Laryngoscope 103 (Suppl 60):29-44, 1993

4. Jackson CG: Glomus tympanicum and glomus jugulare tumors. Otolaryngol Clin North Am 34:941-970, 2001

5. Jackson CG: Neurotologic skull base surgery for glomus tumors. Diagnosis for treatment planning and treatment options. Laryngoscope 103 (Suppl 60):17-22, 1993

6. Jackson CG: Neurotologic skull base surgery for glomus tumors with intracranial extension. Oper Techn Otolaryngol Head Neck Surg 5:112-117, 1994

7. Jackson CG, Netterville JL, Glasscock ME III, et al: Defect reconstruction and cerebrospinal fluid management in neurotologic skull base tumors with intracranial extension. Laryngoscope 102:1205-1214, 1992

8. Jenkins HA, Fisch U: Glomus tumors of the temporal region. Technique of surgical resection. Arch Otolaryngol 107: 209-214, 1981

9. Kinney SE: Glomus jugulare tumors surgery intracranial extension. Otolaryngol Head Neck Surg 88:531-535, 1980

10. Netterville JL, Civantos FJ: Defect reconstruction following neurotologic skull base surgery. Laryngoscope 103 (Suppl 60):55-63, 1993

Manuscript received May 10, 2004.

Accepted in final form July 14, 2004.

Address reprint requests to: C. Gary Jackson, M.D., The Otology Group at Vanderbilt, 300 20th Avenue North, Suite 503, Nashville, Tennessee 37203. email: ozcycle2@aol.com. 